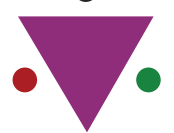

IJCRR

Section: Healthcare Sci. Journal Impact Factor: 6.1 (2018) ICV: 90.90 (2018)

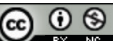

Copyright@IJCRR

\title{
Radiological Assessment of Meniscal Injuries of the Knee on Magnetic Resonance Imaging
}

\section{Shirish V Vaidya ${ }^{1}$, Aneesh M K², Shital M Mahajan³, Harshal S dhongade}

'DMRD, DNB, Assistant Professor, Department of Radiodiagnosis, JNMC, Sawangi, M.S., India; ${ }^{2}$ Professor, Department of Radiodiagnosis, JMMC, Thrissur, Kerala, India; ${ }^{3} \mathrm{MD}$, Assistant Professor, JNMC, Sawangi, M.S., India; ${ }^{M}$ MD Radiodiagnosis, Consultant Radiologist, Apollo Hospital, Nashik, M.S., India.

\section{ABSTRACT}

Background: The anatomical and pathological interpretation of soft tissue, ligaments, fibrocartilage and articular cartilage is superior to that seen with computed tomography (CT) with Magnetic Resonance Imaging (MRI). Fast spin-echo imaging, used for fat suppression in conjunction with MR technique, has improved the sensitivity and accuracy of MR to detect injuries to the articular cartilage. Besides, three-dimensional (3D) volume techniques have shown MR imaging 's flexibility in measuring meniscal tears. This can be used to reformat orthogonal and non-orthogonal representations of meniscal tears planes.

Aim: The present study was done for evaluation of meniscal injuries of the knee on MRI.

Material: This study was carried out in 100 subjects (63 males and 37 females) ageing from 12 to 73 years.

Results: The findings obtained showed that medial meniscal tears/degeneration (64\%) accompanied by anterior cruciate ligament tear (49\%) were the most frequently found pathology of all patients.

Conclusion: Magnetic resonance imaging has a high degree of sensitivity, precision and accuracy in the diagnosis of the meniscal, posterior cruciate ligament and full anterior cruciate tears in detecting partial anterior cruciate ligament tears.

Key Words: Medial meniscus, Lateral meniscus, Magnetic resonance imaging (MRI).

\section{INTRODUCTION}

MRI emerged as the main imaging method in knee joint pathology research. MRI has allowed non-invasive evaluation of the injured knee while preventing invasive procedures and further morbidity. The studies by Kean and co-workers ${ }^{1}$ and Moon and associates ${ }^{2}$ in 1983 were the first to identify MRI 's ability in evaluating the knee joint. In 1985, when MR imaging of the knee first became available, the clinical decision-making process was rarely influenced by its results, the direction of treatment being determined by physical examination and arthrogram. ${ }^{3,4}$ With the advent of improved and sophisticated equipment, today MR imaging is indispensable in the workup of patients with knee disorders. ${ }^{5,6,7}$ At the mid joint level, the medial and lateral menisci are seen with uniform low signal intensity on MRI. The anterior and posterior horns of the lateral meniscus are attached to the nonarticular area of the tibial plateau, contributing to its relatively circular appearance. ${ }^{8.9}$ The lateral meniscus has a more circular shape and consistent width. Sections that are $3 \mathrm{~mm}$ or less in thickness display both menisci on axial images. The anterior and posterior horns of the $\mathrm{C}$-shaped medial meniscus are attached forward on the anterior aspect of the proximal tibia and the posterior tibia above the posterior cruciate attachment, respectively. ${ }^{10,11}$ The medial meniscus has an open Cshaped configuration with a narrow anterior horn and wider posterior horn. On coronal plane images, the body and the anterior and posterior horns of the medial and lateral meniscus are seen as distinct segments and not as opposing triangles as on sagittal images..$^{10}$ On posterior coronal images, the plane of section is parallel with the posterior curve of the $\mathrm{C}$-shaped menisci, and the posterior horn may be seen as a continuous band of low signal intensity.

Aims \& Objectives: Evaluation of meniscal injuries of the knee on MRI.

\section{MATERIAL \& METHODS}

Selection criteria: Patients reporting knee injury history and consenting to the report. Patients assist in the duration of the test (capacity to remain immobile during the evaluation process).

\section{Corresponding Author:}

Dr. Shirish V Vaidya, DMRD, DNB, Assistant Professor, Department of Radiodiagnosis, JNMC, Sawangi, M.S., India Email: drshirish283@gmail.com

ISSN: 2231-2196 (Print)

Received: 02.06 .2020
ISSN: 0975-5241 (Online)

Revised: 11.07 .2020
Accepted: 28.07 .2020 
Exclusion criteria: Patients with heart pacemakers, body metallic sensors, eye or claustrophobic foreign bodies.

MRI scanner: MRI was performed with a PHILIPS ACHIEVA 1.5 T MR Scanner. A dedicated knee coil (FLEX M) was used.

Patient positioning: The procedure was performed after all patients had ruled out the possibility of any metallic implants or prosthesis. The patient was put in a close-coupled extremity coil with the knee in the supine position. The knee was rotated $15-20^{\circ}$ externally, to allow full visualization of the anterior cruciate ligament on sagittal images as it realigns the ACL parallel to the sagittal image axis. The knee was slightly flexed $5-10^{\circ}$ to improve the precision of the patellafemoral compartment and patellar alignment assessment.

Imaging protocol: Standard phased-array surface FLEX M coil for the knee was used routinely. For subsequent sagittal and coronal plane images, an axial acquisition by patellafemoral joint was used as an initial localiser. The coronal plane tests the collateral ligaments and the menisci body in an optimum way. The sagittal plane shows the cruciate ligaments, menisci and synovial anatomy. With the convergence of all three planes the bones, muscles, tendons, neurovascular structures have been thoroughly evaluated.

\section{RESULTS AND OBSERVATION}

In this present study of 100 patients, 63 were men and 37 women. The youngest patient in the study was of $12 \mathrm{yrs}$, while the oldest patient was of 73 yrs. Majority of the patients were in the age group of 21- $50 \mathrm{yrs}$ with those in the age group of 41-50 yrs. being highest. Of the 100 cases, 52 had right-knee derangements while 48 had left-sided derangements. Among 100 patients, 64 had medium meniscal tears and degeneration and 31 had lateral meniscal tears and degeneration.

Table 1: Distribution of Knee Pathologies:

\begin{tabular}{clc} 
Sr. No & Pathology & $\begin{array}{c}\text { No. of } \\
\text { Patients }\end{array}$ \\
\hline 1 & Medial Meniscal Tears & 64 \\
2 & Lateral Meniscal Tears & 31 \\
3 & Anterior Cruciate Ligament Tears & 49 \\
4 & Posterior Cruciate Ligament Tears & 9 \\
5 & Medial Collateral Ligament Tears & 5 \\
6 & Lateral Collateral Ligament Tears & 2 \\
7 & Patellar Subluxations and/or Patellar & 5 \\
8 & Retinaculum Tears & 22 \\
9 & Popliteal Cysts & 12 \\
10 & Osteochondral Fractures & 29
\end{tabular}

Medial meniscal tears \& degeneration: Of the 64 patients with medial meniscal tears and degeneration, 5(7.81\%) showed Grade I signal, 6(9.3\%) showed Grade II signal, 49(76.5\%) showed Grade III tear, 2(3.2\%) showed bucket handle tear and $2(3.2 \%)$ showed root tear. In our study, Grade III tear was the most common.

\begin{tabular}{|c|c|c|}
\hline $\begin{array}{l}\text { Medial Meniscal Tear/ } \\
\text { Degeneration }\end{array}$ & Number & Percentage \\
\hline GRADE I & 05 & $7.81 \%$ \\
\hline GRADE II & 06 & $9 \cdot 3 \%$ \\
\hline GRADE III & 49 & $76.5 \%$ \\
\hline Bucket Handle Tear & 02 & $3.2 \%$ \\
\hline Root Tear & 02 & $3.2 \%$ \\
\hline
\end{tabular}

Distribution of medial meniscal grade III tears: Out of 49 patients of medial meniscal grade III tears, $42(87.75 \%)$ were in posterior horn and $6(12.25 \%)$ were in the anterior horn. Tear involved both anterior and posterior horn in 1 patient.

Table 3: Distribution Of Medial Meniscal Grade III Tears.

\begin{tabular}{lcc} 
Medial Meniscal Grade III Tears & Number & Percentage \\
Anterior Horn & 6 & $12.25 \%$ \\
Posterior Horn & 42 & $85.71 \%$ \\
Both Horns & 1 & $2.04 \%$ \\
\hline
\end{tabular}

Lateral meniscal tears \& degeneration: Out of the 31 cases of lateral meniscal tears and degeneration, 3(9.67\%) showed Grade I degeneration, 12(38.7\%) showed Grade II degeneration and 16(51.61\%) showed Grade III tear. In our study Grade, III Tear (51.61\%) was the most common.

\begin{tabular}{|c|c|c|}
\hline $\begin{array}{l}\text { Lateral meniscal tears/ } \\
\text { degeneration }\end{array}$ & Number & Percentage \\
\hline GRADE I & 3 & $9.67 \%$ \\
\hline GRADE II & 12 & $38.7 \%$ \\
\hline GRADE III & 16 & $51.61 \%$ \\
\hline
\end{tabular}

Distribution of lateral meniscal grade III tears: Out of 16 patients of lateral meniscal Grade III Tears, 7(43.75\%) were in anterior horn and $9(56.25 \%)$ in the posterior horn. In our study, the Lateral Meniscal Grade III tears were more common in posterior horn than in anterior horn. 
Table 15: Distribution of Lateral Meniscal Grade III Tears

\begin{tabular}{lcc} 
Lateral Meniscalgrade III Tears & Number & Percentage \\
Anterior Horn & 7 & 43.75 \\
Posterior Horn & 9 & $56.25 \%$ \\
\hline
\end{tabular}

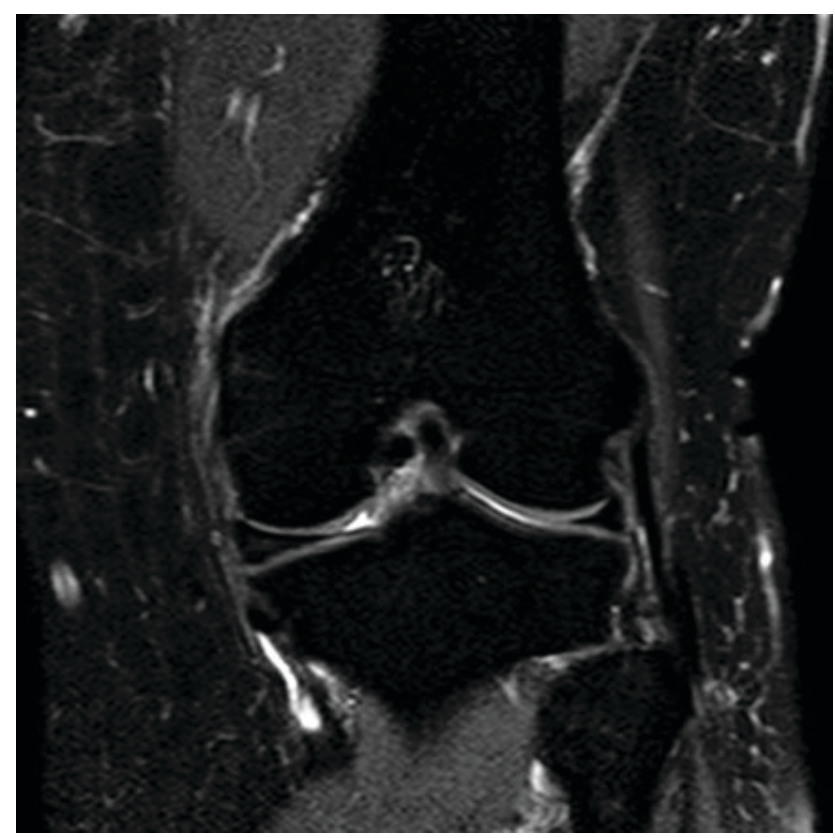

Figure 1: PDFS Coronal Image of Grade I Meniscal Degeneration

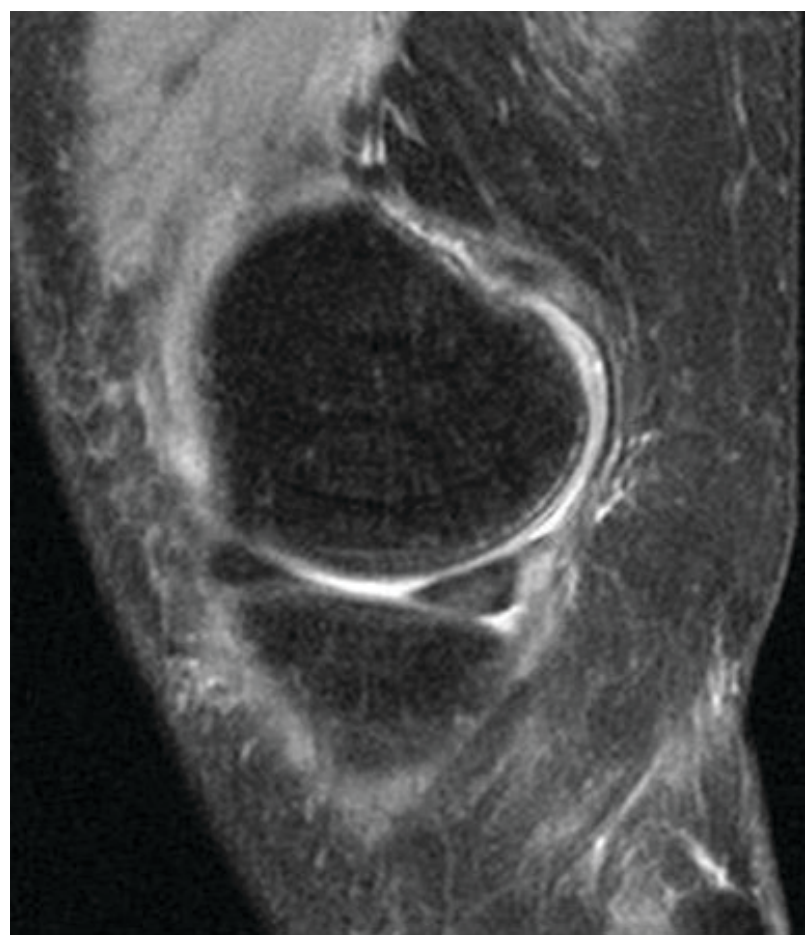

Figure 2: PDFS sagittal Image of Grade II Meniscal Degeneration.

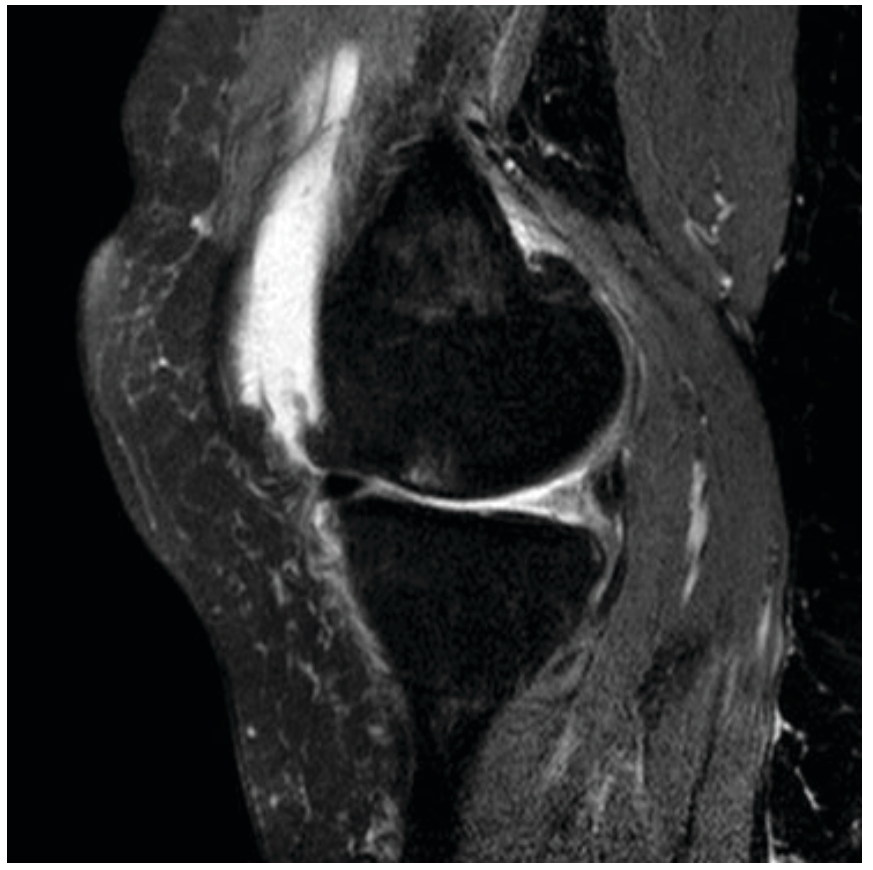

Figure 3: PDFS image showing vertical grade III tears of the medial meniscus

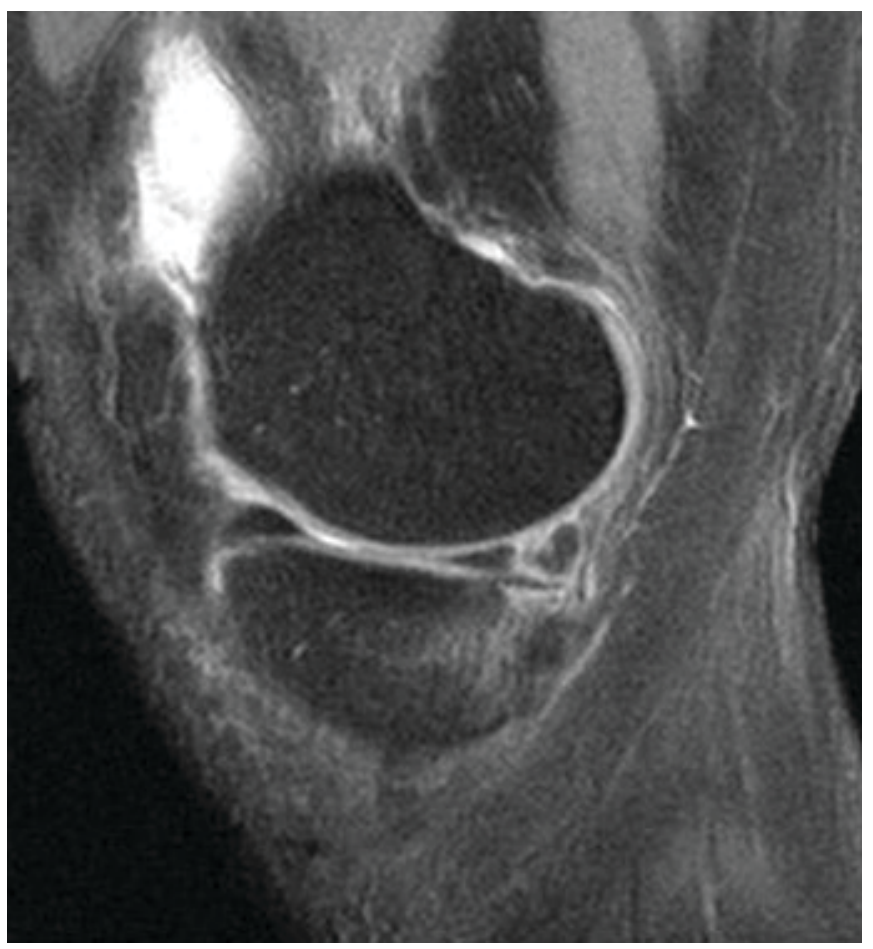

Figure 4: PDFS sagittal image of Medial Meniscal Longitudinal Tear.

\section{DISCUSSION}

Various imaging modalities are used for evaluating knee pathological conditions. The role of MRI in knee imaging has 
gradually increased over the past few years and is now the primary or the only imaging method for evaluating suspected internal derangements. ${ }^{10}$ For traditional and CT arthrography ${ }^{11,12}$, complete evaluation of the capsule, collateral ligaments, menisci, and tendons around the knee was difficult. Multiplanar MR images provide a major improvement in the assessment of these structures. Of 100 patient numbers, 64 (64\%) showed medial meniscal tears and degeneration and 31 (31\%) showed lateral meniscal tears and degeneration. In our sample, medial meniscal tears and degeneration were more frequent (64\%), which is in agreement with Lakhar et al. ${ }^{12}$ who reported $57(38.23 \%)$ cases with medial meniscal tears and 28 cases $(29.41 \%)$ with lateral meniscal tears in their analysis of 173 patients. Shetty et al. ${ }^{14}$ also identified medial meniscal tears as being more common than lateral meniscal tears.

Medial meniscal tears \& degeneration: $5(21.27 \%)$ of the 64 medial meniscal tears and degeneration showed grade I degeneration, 6(17.02\%) showed grade II degeneration, $49(57.44 \%)$ showed grade III tear, $2(2.12 \%)$ showed bucket handle tear, and $2(2.12 \%)$ showed root tear. We found that medial meniscal tears of grade III were more common; however, in their study Stoller et al. ${ }^{15,16 .}$. showed that medial meniscal tears of grade II were more common. Menisci degeneration has demonstrated high signal strength due to impregnated synovial fluid. In our analysis, we found that $\mathrm{T} 2$ * weighted GRE images, as endorsed by Rubin et al. ${ }^{17}$, clearly depicted the meniscal tears than FSE images. Out of 49 patients with grade III medial meniscal damages, 42 (85.71\%) were in the posterior horn, and $6(12.25 \%)$ were in the anterior horn. In 1(2.04 \%) patient, Tear involved both ears. The most common position of medial meniscal tears in our study was at the posterior horn (85.71\%).

Lateral meniscal tears \& degeneration: Of the 31 lateral meniscal tears \& degeneration, $3(9.67 \%)$ showed degeneration in Grade I, 12(38.7 \%) showed degeneration in Grade II and $16(51.61 \%)$ showed Grade III tear. Grade III tears from medial meniscus and lateral meniscus have been the most frequent form of tears among all meniscal tears/degeneration in our research. Stoller et al. ${ }^{15,16}$ recorded the more severe grade II degeneration.

\section{CONCLUSIONS}

In our research, as in other studies, sequences of fast spinecho T2 and proton density FS were found to be most susceptible to the spectrum of meniscal and cruciate ligament tears including related injuries such as osteochondral fractures, whereas the SE T1 sequence was useful for correlating pathology other than ligament tears. PD FS sequences were useful for determining bone contusions associated with it. The high degree of accuracy in viewing MR images and imagery in various planes and placing the knee in $15-20^{\circ}$ of external rotation and $5-10^{\circ}$ of bending helped to delineate the location and the full extent of the lesions. Magnetic resonance imaging has high sensitivity, specificity and accuracy in diagnosing meniscal, posterior cruciate ligament and complete anterior cruciate ligament tears; however, does not have satisfactory sensitivity in detecting partial anterior cruciate ligament tears. Magnetic resonance imaging (MRI) offers ever-increasing diagnostic imaging solutions for knee soft tissue injuries. MRI is the choice of approach for diagnosing and characterizing meniscal and ligamentous knee injuries.

\section{Acknowledgement: None}

Ethical clearance- Taken from the institutional ethics committee.

\section{Source of funding- Self.}

\section{Conflict of Interest- Nil.}

\section{REFERENCES}

1. Kean DM, Worthington BS, Preston BJ. Nuclear MRI of knee: examples of normal anatomy and pathology. Br J Radiol 1983; 56: 355-361.

2. Moon KL, Genant HK, Helms CA, Chafetz NI, Crooks LE, Kaufman L. Musculoskeletal applications of nuclear MR. Radiology 1983; 147: 161-171.

3. Nagrale N, Barmate N. Age determination by radiological assessment of epiphyseal union of bones at knee joint in chhattisgarh region. Journal of South India Medicolegal Association. 2019; 11 (1): 30-35.

4. Mahato R, Panchal D et al. Exercise interventions in Knee Osteoarthritis in Aging adults: A Systematic Review of Randomized Clinical Trials International Journal of Current Research and Review. 2011; 23 (11):01-05.

5. Kursunoglu-Brahme S, Resnick D. Magnetic resonance imaging of the knee. Orthop Clin North Am 1990; 21:561.

6. Mink JH, Deutsch AL. Magnetic resonance imaging of the knee. Clin Orthop 1989; 244:29.

7. Burk DL Jr, Mitchell DG, Rifkn MD, Vinitski S. Recent advances in magnetic resonance imaging of the knee. Radiol Clin North Am 1990; 28:379

8. Johnson RL. Anatomy and biomechanics of the knee. In: Chapman MW, ed. Operative orthopaedics, 2nd ed. Philadelphia: JB Lippincott, 1993:2039.

9. Kumar P, Kumar A et al. Healthy Gait: Review of Anatomy and Physiology of Knee Joint International Journal of Current Research and Review. 200312 (3): 01-08.

10. Gray SD, Kaplan PA, Dussalt RG. Imaging of knee: current status. Orthopaedics Clinics of North America 1997; 28 (4): 643658.

11. Gray H, Williams PL, Bannister LH. Gray's anatomy: the anatomical basis of medicine and surgery, $38 \mathrm{t}^{\mathrm{th}}$ edition $1995 ; 678$ 712.

12. B N Lakhkar, K V Rajagopal, P Rai. MR Imaging of Knee with Arthroscopic Correlation in Twisting Injuries. Ind J Radiol Imag. $2004 ; 14: 1: 33-40$. 
13. La Prade RF, Burnett QM, Veenstra MA, Hodgman CG. The prevalence of abnormal MRI findings in asymptomatic knees. Am J Sp Med 1994; 171: 761-766.

14. D S Shetty, B N Lakhkar, G K Krishna. Magnetic Resonance Imaging in Pathologic Conditions of Knee. Ind J Radiol Imag 2002; $12: 3: 375-381$.

15. Stoller DW, et al. Meniscal tears: pathological correlation with MR imaging. Radiology 1987; 163:731-735.
16. Stoller DW, et al. Meniscal tears: pathological correlation with MR imaging. Radiology 1987; 163:731-735.

17. Rubin DA, Kneeland JB, Listerud J, Underberg E, Davis SJ. MR diagnosis of meniscal tears of the knee: value of FSE vs conv SE pulse sequences. AJR 1994; 162: 1131-1138. 